\title{
Magnetohydrodynamic equilibria in barotropic stars
}

\author{
C. Armaza ${ }^{1}$, A. Reisenegger ${ }^{1}$, J. A. Valdivia ${ }^{2}$ and P. Marchant ${ }^{1,3}$ \\ ${ }^{1}$ Instituto de Astrofísica, Facultad de Física, Pontificia Universidad Católica de Chile, \\ Av. Vicuña Mackenna 4860, 782-0436 Macul, Santiago, Chile \\ email: cyarmaza@uc.cl \\ ${ }^{2}$ Departamento de Física, Facultad de Ciencias, Universidad de Chile, \\ Casilla 653, Santiago, Chile. \\ ${ }^{3}$ Argelander Institut für Astronomie, Universität Bonn, \\ Auf dem Hügel 71, D-53121, Bonn, Germany
}

\begin{abstract}
Although barotropic matter does not constitute a realistic model for magnetic stars on short timescales, it would be interesting to confirm a recent conjecture that states that magnetized stars with a barotropic equation of state would be dynamically unstable (Reisenegger 2009). In this work we construct a set of barotropic equilibria, which can eventually be tested using a stability criterion. A general description of the ideal MHD equations governing these equilibria is summarized, allowing for both poloidal and toroidal magnetic field components. A new finite-difference numerical code is developed in order to solve the so-called Grad-Shafranov equation describing the equilibrium of these configurations, and some properties of the equilibria obtained are briefly discussed.
\end{abstract}

Keywords. MHD, stars: magnetic field

\section{Overview}

The persistence of magnetic fields in massive stars and their stellar remnants motivates the study of what physical conditions are involved in sustaining such configurations. In this context, barotropic equations of state, where pressure is a function solely of density, are often assumed to describe the matter within these objects (Yoshida \& Eriguchi 2006; Haskell et al. 2008; Lander \& Jones 2009; Ciolfi et al. 2009; Fujisawa et al. 2012). Barotropy strongly restricts the range of possible equilibrium configurations and does not strictly represent the realistic stably stratified matter within these objects, which is likely to be an essential ingredient in the stability of magnetic fields in stars on short timescales (Reisenegger 2009).

The question whether magnetic equilibria in barotropic stars can be stable or not remains as an important issue to be answered. Several authors (Lander \& Jones 2009; Ciolfi et al. 2009; Fujisawa et al. 2012; Gourgouliatos et al. 2013) have explored the possible axially symmetric equilibria in barotropic stars, generally finding that the fraction of the total magnetic energy corresponding to the toroidal component, $E_{\text {tor }} / E_{\text {mag }}$, is at most a few \%. In addition to not being enough to account for the energy emitted by magnetars, this would be insufficient to stabilize the poloidal component (Braithwaite 2009; Akgün et al. 2013), as confirmed by the simulations of Lander \& Jones (2012). However, recent simulations (Ciolfi \& Rezzolla 2013; Fujisawa \& Eriguchi 2013) have shown that higher fractions $E_{\text {tor }} / E_{\text {mag }}$ are possible, making a more extensive survey of these equilibria relevant. Studying properties of barotropic equilibria could be also relevant considering the scenario in which neutron stars would reach an effectively barotropic 
state after overcoming stable stratification by means of direct and inverse $\beta$-decays and ambipolar diffusion acting on timescales shorter than their lifetime (Hoyos et al. 2008; Reisenegger 2009; Reisenegger, these Proceedings; Mitchell et al., these Proceedings).

This ongoing research is focused on obtaining a wide range of numerical barotropic equilibria, paying attention to their main properties. In addition, these results can be considered as a starting point to study in more detail whether magnetic fields in a wide range of barotropic equilibria are stable or not (Mitchell et al., these Proceedings).

\section{Barotropic equilibria: the Grad-Shafranov equation}

Throughout this work, we take the approach of considering a magnetic star within the context of ideal MHD, that is, a perfectly conducting fluid in dynamical equilibrium described by the Euler equation,

$$
\nabla P+\rho \nabla \Phi=\frac{1}{c} \mathbf{J} \times \mathbf{B},
$$

where the right-side is the Lorentz force per unit volume. All known stars have a very large fluid pressure $P\left(P \sim G M^{2} / R^{4}, M\right.$ being the mass and $R$ the radius $)$, to magnetic pressure $B^{2} / 8 \pi$ ratio ( $B$ being an estimation of the maximum magnetic field strength), $8 \pi P / B^{2} \gtrsim 10^{6}$ (Reisenegger 2009), which suggests that magnetic fields do not play an important role in the structure of these stars, so at first approximation we can consider the star as spherical, with negligible deformations due to magnetic forces. In addition, if axial symmetry is assumed, and spherical coordinates $(r, \theta, \phi)$ are used to describe the model, all scalar quantities are independent of the azimuthal coordinate, and the magnetic field may be expressed as the sum of a poloidal (meridional) component, and a toroidal (azimuthal) component, each determined by a single scalar function,

$$
\mathbf{B}=\mathbf{B}_{\mathrm{pol}}+\mathbf{B}_{\mathrm{tor}}=\boldsymbol{\nabla} \alpha(r, \theta) \times \boldsymbol{\nabla} \phi+\beta(r, \theta) \boldsymbol{\nabla} \phi,
$$

which turn out to be constant along their respective field lines (Chandrasekhar \& Prendergast 1956). Under this symmetry, the azimuthal component of the magnetic force per unit volume must vanish, which implies a functional relation between these scalar functions, $\beta(r, \theta)=\beta(\alpha(r, \theta))$. In this way, both $\alpha$ and $\beta$ are constant along field lines and, if a vacuum is assumed outside the star, the toroidal field may lie only in regions where the poloidal field lines close within the star. On the other hand, if a barotropic equation of state, $P=P(\rho)$, is assumed, the Lorentz force per unit mass must be the gradient of some arbitrary function $\chi(r, \theta)$, which turns out to be a function of $\alpha$ as well, $\chi(r, \theta)=\chi(\alpha(r, \theta))$. From this, a non-linear elliptic partial differential equation is found to be the master equation governing barotropic MHD equilibria, the so-called Grad-Shafranov (GS) equation,

$$
\frac{\partial^{2} \alpha}{\partial r^{2}}+\frac{\sin \theta}{r^{2}} \frac{\partial}{\partial \theta}\left(\frac{1}{\sin \theta} \frac{\partial \alpha}{\partial \theta}\right)+\beta \beta^{\prime}+r^{2} \sin ^{2} \theta \rho \chi^{\prime}=0
$$

(Grad \& Rubin 1958, Shafranov 1966), where primes stand for derivatives with respect to the argument, and both $\beta=\beta(\alpha)$ and $\chi=\chi(\alpha)$ are arbitrary functions, whose form may be chosen depending on the particular magnetic configuration of interest. Under the assumption of weak magnetic field discussed in this section, the density $\rho$ appearing in the GS equation may be replaced by its non-magnetic background counterpart, $\rho=\rho(r)$, such that we solve for the magnetic functions for a given density profile, instead of considering the more difficult task of solving self-consistently for the magnetic functions and for the fluid quantities, as done, e. g., by Lander \& Jones (2012). 

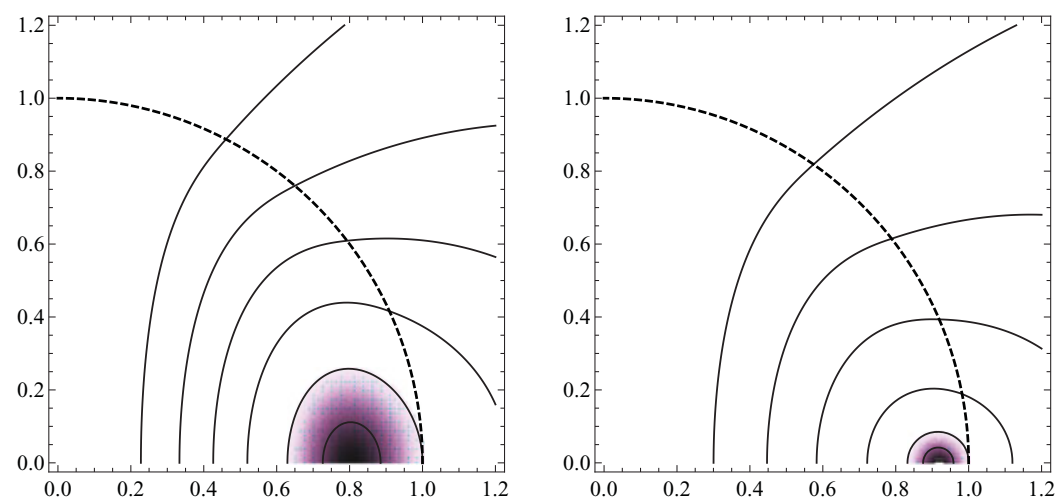

Figure 1. Numerical equilibria found using our code. In both cases, we used $\beta(\alpha)$ as given in Eq. (3.1), together with $\chi(\alpha)=\alpha$. Left: $s=10$, right: $s=45$. The dashed line corresponds to the stellar surface. Poloidal field lines are shown in solid black curves while the color map accounts for $\left(r \sin \theta B_{\phi}\right)$.

\section{Numerical solutions and discussion}

In order to obtain suitable barotropic equilibria, we have developed a finite-difference code to solve numerically the GS equation inside the star, for arbitrary choices of $\beta(\alpha)$, $\chi(\alpha)$ and density profile $\rho(r)$. Outside the star, the function $\alpha(r, \theta)$ may be written as a superposition of multipoles, corresponding to the general solution of the GS equation with both $\beta=0$ and $\rho=0$. Solutions found inside the star are matched to the exterior expansion by demanding continuity of the magnetic field components, in order to avoid surface currents. After testing our code, we studied the so-called twisted-torus configuration

$$
\beta(\alpha)= \begin{cases}s\left(\alpha-\alpha_{s}\right)^{1.1} & \alpha_{s} \leqslant \alpha \\ 0 & \alpha<\alpha_{s},\end{cases}
$$

where $s$ is a free parameter accounting for the relative strength between the poloidal and the toroidal component. Here, $\alpha_{s} \equiv \alpha(R, \pi / 2)$ stands for the value of $\alpha$ along the longest poloidal field line closing within the star, $R$ being the stellar radius, so the toroidal field lies in the region $\alpha(r, \theta) \geqslant \alpha_{s}$ only, as seen in Figure 1. This choice of $\beta(\alpha)$, along with $\chi(\alpha)=\alpha$, allows us to compare with previous works which have taken these same functional forms. For the simple, but reasonably realistic profile $\rho(r)=\rho_{c}\left(1-r^{2} / R^{2}\right)$, we explored the behavior of such configurations by changing the parameter $s$. Figure 2 shows the magnetic field profile for two different values of $s$ : the larger the value of $s$, the stronger the toroidal field becomes, although the region where the toroidal field lies shrinks, as shown in Figure 1.

In all cases, the energy stored in the toroidal component is only a few percent of the total magnetic energy, even when the toroidal field strength is comparable to the poloidal one, in agreement with previous works. Furthermore, this ratio seems to be bounded by a certain maximum value as seen in Figure 3 (left panel), above which the ratio would decrease, as already pointed out in the literature (Lander \& Jones 2009, Ciolfi et al. 2009, Fujisawa et al. 2012), although such a behavior for large $s$ and density profile considered here is not reached in our calculations. It is interesting to see, however, that we do reach a maximum in the ratio of toroidal to poloidal flux (Figure 3, right panel). 

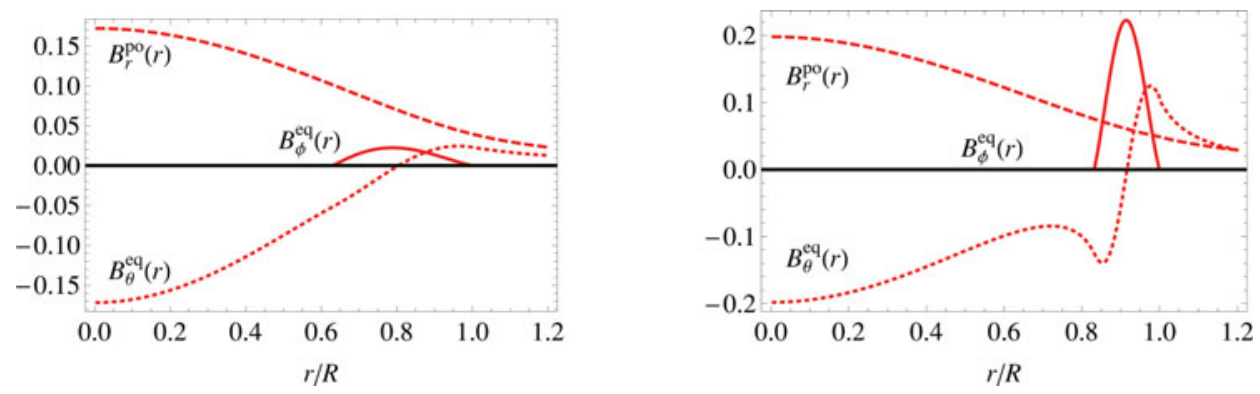

Figure 2. Magnetic field profiles for equilibria in Fig. 1. Left: $s=10$, with $E_{\mathrm{tor}} / E_{\mathrm{mag}} \approx 0.5 \%$. Right: $s=45$, with $E_{\text {tor }} / E_{\mathrm{mag}} \approx 3.5 \%$.
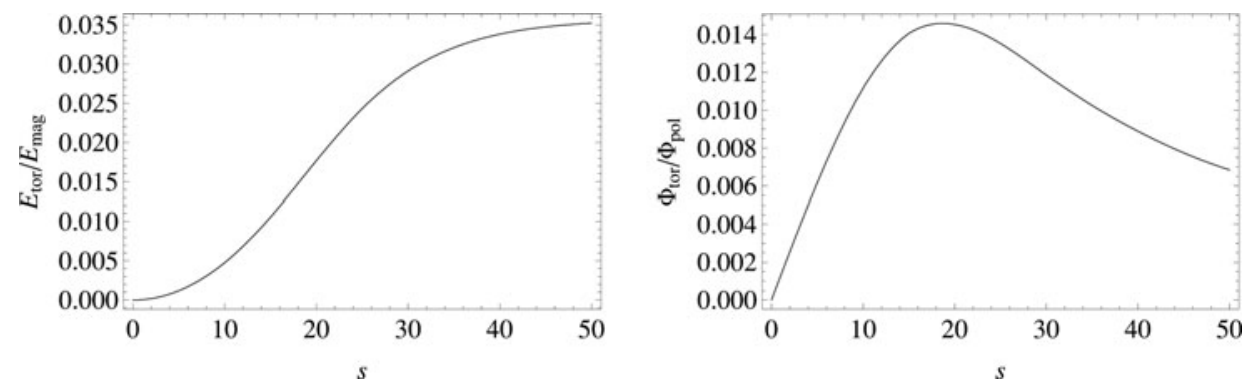

Figure 3. Toroidal-to-total magnetic energy (left) and maximum toroidal-to-maximum poloidal flux (right) ratios as functions of $s$, using $\rho(r)=\rho_{c}\left(1-r^{2} / R^{2}\right)$ and $\chi^{\prime}=1$.

\section{Acknowledgments}

All authors are supported by CONICYT International Collaboration Grant DFG-06. CA and PM have been supported by CONICYT Master's Fellowships. AR and JAV are supported by FONDECYT Regular Grants 1110213 and 1110135, respectively. CA, AR and PM are supported by the Basal Center for Astrophysics and Associated Technologies. CA thanks K. N. Gourgouliatos and K. Fujisawa for very stimulating discussions.

\section{References}

Akgün, T., Reisenegger, A., Mastrano, A., \& Marchant, P. 2013, MNRAS, 433, 2445

Braithwaite, J. 2009, MNRAS, 397, 763

Chandrasekhar, S. \& Prendergast, K. H. 1956, Proc. Nat. Acad. Sci., 42, 5

Ciolfi, R., Ferrari, V., Gualtieri, L., \& and Pons, J. A. 2009, MNRAS, 397, 913

Ciolfi, R. \& Rezzolla, L. 2013, MNRAS, 435, L43

Fujisawa, K., Yoshida, S., \& Eriguchi, Y. 2012, MNRAS, 422, 434

Fujisawa, K. \& Eriguchi, Y. 2013, MNRAS, 432, 1245

Grad, H. \& Rubin, H. 1958, in Proc. 2st Int. Conf. on Peaceful Uses of Atomic Energy. United Nations, Geneva, 31, 190

Gourgouliatos, K. N., Cumming, A., Reisenegger, A., Armaza, C., Lyutikov, M., \& Valdivia, J. A. 2013, MNRAS, 434, 2480

Haskell, B., Samuelsson, L., Glampedakis, K., \& Andersson, N. 2008, MNRAS, 385, 531

Hoyos, J., Reisenegger, A., \& Valdivia, J. A. 2008, A\& $A$, 487, 789

Lander, S. K. \& Jones, D. I. 2009, MNRAS, 395, 2162

Lander, S. K. \& Jones, D. I. 2012, MNRAS, 424, 482

Reisenegger, A. 2009, A\& A, 499, 557

Shafranov, V. D. 1966, in: Reviews of Plasma Physics, (New York: Cons. Bureau), 2, 103

Yoshida, S. \& Eriguchi, Y. 2006, ApJS, 164, 156 